\title{
Word Choice in Mathematical Practice: A Case Study in Polyhedra
}

\author{
Lowell Abrams, ${ }^{\star}$ Landon D. C. Elkind ${ }^{\star \star}$
}

Accepted 2019-06-09, forthcoming

\begin{abstract}
We examine the influence of word choices on mathematical practice, i.e. in developing definitions, theorems, and proofs. As a case study, we consider Euclid's and Euler's word choices in their influential development and, in particular, their use of the term 'polyhedron'. Then, jumping to the 20th century, we look at word choices surrounding the use of the term 'polyhedron' in the work of Coxeter and of Grünbaum. We also consider a recent and explicit conflict of approach between Grünbaum and Shephard on the one hand and that of Hilton and Pedersen on the other, elucidating that the conflict was engendered by disagreement over the proper conceptualization, and so also the appropriate word choices, in the study of polyhedra.
\end{abstract}

Keywords mathematical practice, word choice, polyhedron, history of mathematics, philosophy of mathematics

\section{Introduction}

Mathematics is typically developed and conveyed in language. Most mathematicians know they must choose terms and formulate both definitions and theorems carefully so as to accurately communicate their conceptualization of a mathematical subject-matter. What is less clear is the extent to which this reliance on language influences the actual development of a subject-matter in mathematical practice.

As a case study in the influence of language on mathematical practice, we examine the term 'polyhedron' and consider how the word choices one uses in developing a mathematical subject-matter can (and do) embody distinct (and sometimes divergent) conceptualizations. We take the case of word choices used in the history and development of 'polyhedron' as a fascinating exemplar of the non-trivial role language plays in the development of mathematical subject-matter - a role that we note it necessarily plays so long as language is the primary means of one's mathematical practice. Indeed, drawing the conclusions of his study of a programmatic effort to express mathematics in Maori, Barton tells us that

mathematics arises after, not before, human activity. The development of mathematical language is consistent with the idea that mathematical concepts, objects, and relationships arise through language, and within particular sociocultural environments, in response to human thinking about quantity, relationships, and space. [1, p. 88]

Our analysis of word choice in the context of the development of the notion of polyhedron will in fact reveal different mathematicians emphasizing the perspectives of quantity, relationships, and space differently.

\footnotetext{
* George Washington University, University Writing Program and Department of Mathematics, Ames Hall, Room 207, 2100 Foxhall Road NW, Washington, DC 20007, labrams@gwu.edu

$\star \star$ University of Iowa, Department of Philosophy, English-Philosophy Building, Room 570, 251 West Iowa Avenue, Iowa City, IA 52242, ORCid 0000-0003-0513-2937, landon-elkind@uiowa.edu
}

Address(es) of author(s) should be given 
We focus on word choice as one aspect of language's non-trivial influence on mathematical practice. We do not think, and do not claim, that this is the only way in which language non-trivially influences mathematical practice. Lakoff and Nuñez, for instance, have developed an extensive theory explaining the ways in which metaphor has played, and continues to play, a critical role in the development of mathematics by providing the means to concretize, at least linguistically, the abstract. [13]

Of course, nonlinguistic factors also influence mathematical cognition and thought processes. Manifestly, mathematics, like other institutions, has a history and culture that powerfully shape the direction of mathematical research. Determining to what extent each of these influences mathematical developments, and even disentangling the distinct features of mathematical practice, is not our present concern. As such, we make no conjecture as to if and how word choice determines mathematical developments. Our case study is presented as neutral between competing global accounts of mathematical practice: it is even consistent with the data examined in our case study of word choice that other aspects of mathematical practice explain the influence of word choices in developing a mathematical subject-matter.

That said, even without embracing here a taxonomy of the means through which mathematical practice shapes mathematics, we claim what is supported by our case study of 'polyhedra', that word choice plays a detectable and significant role in mathematical developments. The denial that word choice is influential in mathematics is inconsistent with the data examined in this case study. With this exception, various accounts of what influences word choice, and even divergent global accounts of mathematical practice, are consistent with the data presented here: we show merely that word choice influences mathematics.

For all that, word choice as a critical feature of mathematical practice is understudied. ${ }^{1}$ Hence the need for our case study of 'polyhedra' supporting that word choice influences mathematical practice. This claim, however, needs some clarification before its truth-conditions are comprehensible. To that end, we first explain (although do not define) some vital terms in our case study - word choice, conceptualization, encoding, mathematical subject-matter, and influencing. ${ }^{2}$

By word choice, we mean the (written) words used to develop a mathematical subject-matter. ${ }^{3}$ For example, in presenting set theory one might describe sets as 'containing' their elements, or one might use 'including' them. These are two different word choices that one might use to develop set theory. Word choices have the virtue that they are observable: they occur as concrete objects on a page.

Authorial intent, in contrast, is not observable and word choices may be a misleading guide to authorial intent. Indeed, word choices can misrepresent authorial intent. For example, a mathematician may not intend to communicate about sets as spatial in any way. But a reader may (mis) understand their talk of sets 'containing' elements as a spatial metaphor. Because authorial intent can be imperfectly reflected in word choice, here we avoid consideration or reconstruction of (unobservable) authorial intent, we prefer to speak instead of conceptualizations of mathematical subject-matter encoded by word choice.

By a conceptualization of a mathematical subject-matter, we mean an understanding of some mathematics. The nature of mathematical understanding is controversial. For example, one might view understanding as a mental content one masters and has 'in their head', or in contrast as a social, cultural, and linguistic practice in which one trains and engages. We do not propose a definition of mathematical understanding here: we keep our case study neutral as between divergent accounts of it. This much is assumed about mathematical understanding in our case study: an understanding of mathematics is, at minimum, the kind of thing an author, whether intentionally or accidentally, imparts to or trains in their reader by communicating a mathematical subject-matter in (usually written) words.

Methodologically, we assume that words can communicate a conceptualization of a mathematical subject-matter with varying degrees of success. If words are transparent, so that the conceptualization is clear and distinct, then this is the highest degree of success. But words might communicate a conceptualization of a mathematical subject-matter less well, just as 'containment' may misleadingly communicate a spatial conceptualization of sets. Assuming that words can communicate a conceptualization more or less well, we can speak of word choices encoding a conceptualization of a mathematical subject-matter.

By word choices encoding a conceptualization of a mathematical subject-matter, we mean that word choices communicate some (perhaps more than one) conceptualization of the subject-matter (which

\footnotetext{
1 A search of "word choice" and "mathematical practice" returns only six articles, all of which are in education studies. A search for "word choice" and "mathematics" returns only 29 works, mostly in education studies and some in linguistics.

2 We thank an anonymous reviewer for inviting us to state and clarify our methodological assumptions.

3 We do not discuss here spoken mathematics or visual mathematics. But we conjecture that our remarks about word choices in written mathematics also apply to those in spoken mathematics and to diagram choices in visual mathematics.
} 
can be independent of, in harmony with, or contrary to, what the author intends to communicate). The evidence that word choices do this at all is to be found by considering how the conceptualization encoded by word choice influences mathematical practice, i.e. the development of a mathematical subject-matter.

By a mathematical subject-matter, we mean to include its objects of study, theorems, constructions, examples, proofs, methods, and informal framing remarks. ${ }^{4}$ It is controversial whether a subject-matter also needs some kind of foundation or internal unity; it is also controversial whether mathematical subjectmatters are amenable to sharp delimitation. ${ }^{5}$ The precise nature of these things - e.g. the ontology of mathematical objects, what makes a string of symbols a proof, and so on - is also controversial. Our case study leaves these issues open. But what we mean by 'the development of these things' in a text (e.g. an article or a book) is comprehensible even though the nature of the 'things' is controversial. The development of a mathematical subject-matter is what we call mathematical practice.

By something's influence on mathematical practice, we mean to include how it expands or restricts the range of objects in the domain of inquiry, how it facilitates or hinders the statement or discovery of theorems, and how it makes available or eases constructions or proofs. ' $X$ influences mathematical practice' means $X$ influences (in the specified ways or others) the actual development of a mathematical subject-matter. In this case study of 'polyhedra', we examine and show how word choice does just this.

In Section 2, we review the treatment of polyhedra by Euclid and Euler to illustrate the impact of word choice on the development of mathematical results. In Section 3 we turn to two book-length texts, Regular Polytopes by H. S. M. Coxeter and Convex Polytopes by B. Grünbaum, to examine the role of language choices in mathematical exposition. We conclude Section 3 on a connection with D. Reed's work, which highlights an aspect of the connection between mathematics and meta-mathematics, namely how a text delimits a mathematical investigation. In Section 4, we discuss some thoughts from I. Lakatos on formalizing mathematical ideas, then we describe a recent disagreement regarding how to extend Euler's famous counting formula $V-E+F=2$, between Grünbaum and Shephard on the one hand and Hilton and Pedersen on the other. We show that this conflict partly rests on the issues of language and word choice raised in Sections 2 and 3. In Section 5, we draw out some of the lessons of our study.

\section{Word Choice and Conceptions of Polyhedra}

We examine Euclid and Euler (in that order) to show that word choice conveys different conceptions of solids and also that different notions of an object of study can lead to different mathematical results. ${ }^{6}$ We summarize our findings at the end of this section.

\subsection{Euclid's Elements}

We note first that in our treatment of Euclid, distinguishing between a motion and an action is important. A motion is a specified operation upon some object or objects resulting in some change. An action is some change enacted upon an object or objects without detailing explicitly how that change occurs. Both motion and action may involve motion-language, but in Euclid motions necessarily do while actions need not. Falling is an instance of motion. Separating is an example of an action. The former specifies how the end result is achieved - passing through a spatial region - whereas the latter does not. Euclid invokes both motion and action, but as we will see, Euclid's word choice encodes a conception of polyhedra on which motion-language is critical to his construction of polyhedra. ${ }^{7}$

Euclid employs motion-language to communicate his mathematics. Consider, for example, his definition of a circle: "A circle is a plane figure contained by one line such that all the straight lines falling upon it from one point..." $\left[4\right.$, Book 1, Definition 15] ${ }^{8}$ His definition of a circle utilizes the motion-language of falling. The conception of geometric objects thereby communicated is one of moving objects about an

\footnotetext{
4 We do not explain what makes a subject-matter mathematical on the assumption that any plausible account of this would have as a consequence that solid geometry, including the study of polyhedra, is a mathematical subject-matter.

5 Confer [14, Introduction, esp. §2, and Chs 6 and 7].

6 Different notions may also result in the same theorems while developing a subject by distinct paths.

7 We thank Michael Blaustein of St. John's College for pointing out to Abrams the importance of motion in Euclid's work.

8 When we quote Euclid, we use the standard Thomas L. Heath translation. Emphasis and brackets come from the original text. We do rely on translations alone in our discussions of Greek and Latin texts.
} 
ambient space. Thus, even in examples such as "A circle does not cut a circle at more points than two" [4, Book 3, Proposition 10], the action implicitly relies on motion-language of cutting, so that one circle is said to "cut" another. The conceptualization of geometric objects as given through an ambient space is further evidenced by the definition of circle invoking containment by a line, as part of a plane figure, thus conceiving of a circle as what has been spatially surrounded. In contrast, talk about set-theoretic containment, meaning set-membership, does not critically rely on motion-language.

Euclid's first postulate similarly utilizes motion-language: "To draw a straight line from any point to any point." [4, Book 1, Postulate 1] This postulate involves the motion of drawing a line through space between two part-less occupants of that space.

Propositions of the Elements also incorporate motion-language, as here in the antecedent of a theorem mid-way through Book 1: "If a straight line falling on the two straight lines..." [4, Book 1, Proposition 27] This ubiquitous conceptualization of geometry as a spatial endeavor grounds the development of Euclid's geometry, and is manifested in Euclid's mathematical practice in his word choices.

As our guiding interest here is in polyhedra, we skip to the later sections of the Elements to establish that Euclid communicates his solid geometry using spatial metaphors, including motion-language. Consider Euclid's construction of a tetrahedron:

If then, $K L$ remaining fixed, the semicircle be carried round and restored to the same position from which it began to be moved, it will also pass through the points $F, G, \ldots[4$, Book 13, Proposition $13]$

Motion language cannot be removed from Euclid's proof because Euclid cannot refer to a single semicircle being carried around if that very semicircle is not being moved. So Euclid's choice of words, including "carrying" and "passing through," communicates conceptual and mathematical content necessary for his argument to go through. Without it, his words communicate a gap-ridden proof at best, for without such motion-language, we cannot readily describe the construction that Euclid means to communicate, despite our being able to offer a wholly different construction that does not appeal to motion-language.

Some of Euclid's definitions for his solid geometry also utilize motion-language:

The inclination of a straight line to a plane is, assuming a perpendicular drawn from the extremity of the straight line which is elevated above the plane to the plane, and a straight line joined from the point thus arising to the extremity of the straight line which is in the plane, the angle contained by the straight line so drawn and the straight line standing up. [4, Book 11, Definition 5]

The completed motions here - "so drawn" and "[so] standing up" - communicate the concept demarcated in Definition 5 of Book 11. Euclid is not just augmenting the reader's informal understanding of his definition; drawing and standing, completed motions, are constitutive of his definition of the notion of the inclination of a straight line to a plane. ${ }^{9}$

Euclid again employs motion-language in Proposition 18 of Book 13, "To set out the sides of the five figures and to compare them with one another." The construction begins:

Let $A B$, the diameter of the given sphere, be set out, and let it be cut at $C$ so that $A C$ is equal to $C B$, and at $D$ so that $A D$ is double of $D B$; let the semicircle $A E B$ be described on $A B$. From $C D$, let $C E, D F$, be drawn at right angles to $A B$, and let $A F, F B, E B$ be joined. [4, Book 13, Proposition 18]

It is on the basis of motion-language, like "joined", "set out", "cut", and "drawn" that Euclid communicates his notions. It is through motion-language word choices that Euclid compares the sides of solid figures. Compare this conceptualization with the modern notion of a metric, which tends not to be communicated in motion-language.

Motion-language is also the bridge between Euclid's two-dimensional geometry and his three-dimensional one; for Euclid defines and constructs three-dimensional objects as completed motions of two-dimensional objects. Consider Euclid's definition of a sphere:

When, the diameter of a semicircle remaining fixed, the semicircle is carried round and restored again to the same position from which it began to be moved, the figure so comprehended is a sphere. [4, Book 11, Definition 14]

\footnotetext{
9 As logicians might put it, Euclid's meta-theoretic language is the language of motion.
} 
Completed motions of a semicircle constitute a sphere: for Euclid, a sphere is a figure enclosed by the completed motion of a semicircle. ${ }^{10}$ As another example, take Euclid's construction of a tetrahedron:

To construct a pyramid, to comprehend it in a given sphere...Let the diameter $A B$ of a given sphere be set out... from the point $H$ let $H K$ be set up at right angles to the plane...therefore the semicircle described on $K L$ will pass through $E$ also. If then, $K L$ remaining fixed, the semicircle be carried round and restored to the same position from which it began to be moved, it will also pass through the points $F, G \ldots[4$, Book 13, Proposition 13]

All these steps either involve or result from completed motions, as in laying out the diameter or drawing on a line. The construction begins by enclosing a space within a sphere, and the study of the tetrahedron relies on this spatial enclosure. Euclid studies the completed tetrahedron by encompassing it spatially; for him, this mathematical task is a spatial one.

In the section that immediately follows Proposition 18's proof, Euclid proves that only five regular solids can be constructed. ${ }^{11}$ For Euclid, this assertion involves the spatial notion of containment: "I say next that no other figure, besides the said five figures, can be constructed which is contained by equilateral and equiangular figures equal to one another." [4, Book 13] Euclid's proof exhausts the possible arrangements of two-dimensional components of solid angles. But motion-language occurs here as elsewhere: "...but a solid angle cannot be formed by six equilateral triangles placed together at one point..." [4, Book 13] ${ }^{12}$ Here the claim is that we cannot "place together" equilateral triangles at a point to form a solid angle. An inability to construct a solid figure is equated with the impossibility of placing together two-dimensional figures, and so the proof of Euclid's capstone result utilizes motion-language.

We close this section by noting that Euclid's motion-language is part-and-parcel of a conception of polyhedra on which a polyhedron is a region of a pre-given (unconstructed) ambient space. Euclid's motion-language relies on there being such an ambient space to move objects through. For example, his definition of solid angle presumes that there is a space such that multiple lines drawn through it can consequently contain or bound regions, which are then defined to be solids in the Elements:

A solid angle is the inclination constituted by more than two lines which meet one another and are not in the same surface, towards all the lines. Otherwise: A solid angle is that which is contained by more than two plane angles which are not in the same plane and are constructed to one point. [4, Book 11, Definition 11]

A solid is that which has length, breadth, and depth... Similar solid figures are those contained by similar planes equal in multitude... When, the diameter of a semicircle remaining fixed, the semicircle is carried round and restored again to the same position from which it began to be moved, the figure so comprehended is a sphere. [4, Book 11, Definitions 1, 9, 14]

Euclid defines the notion of similarity in terms of the figures that bound the spatial regions that solids are said to be. The solid itself, the spatial region, is constructed (bounded) by a motion that cordons off part of the ambient space. That is how he defines the pyramid and the sphere. Euclid's definitions of the Platonic solids are likewise given in terms of figures that, when they are moved about the ambient space, consequently bound a spatial region:

A pyramid is a solid figure, contained by planes, which is constructed from one plane to one point... A cube is a figure contained by six equal squares. An octahedron is a solid figure contained by eight equal and equilateral triangles. An icosahedron is a solid figure contained by twenty equal and equilateral triangles. A dodecahedron is a solid figure contained by twelve equal, equilateral, and equiangular pentagons [4, Book 11, Definitions 12 and 25-28].

To summarize, Euclid defines, studies, and categorizes polyhedra using motion-language. A polyhedron, as Euclid conceives of it, is a region cordoned from the ambient space by the trace of two-dimensional

\footnotetext{
10 Note that a figure is any thing "contained by any boundary or boundaries," another use of spatial containment to communicate Euclid's geometry [4, Book 1, Definition 14].

11 Heath assigns no number to the proposition that only five regular solids can be constructed, preventing us from using the briefer 'Proposition 19'.

12 In fact, Euclid proves that only five regular solids exist by showing that only five combinations of triangles or pentagons result in a solid angle: "For a solid angle cannot be constructed with two triangles, or indeed planes. With three triangles the angle of the pyramid is constructed... but a solid angle cannot be formed by six equilateral triangles placed together at one point..." [4, 1, p. 480]
} 
figures moved through that space. Note, therefore, that Euclid's mathematical language and word choice are nonsensical if we distinguish a region of space from the polyhedron itself as he conceives of it.

Euclid's word choice ties his conception of polyhedra into his mathematical practice - his definitions, theorems, constructions, and proofs. Let us consider other examples of this phenomenon.

\subsection{Euler's "Proof of Some Notable Properties with which Solids Enclosed by Plane Faces are Endowed"}

In contrast to Euclid, Euler's conception of polyhedra requires treating polyhedra as bodies with structural features that are separable from the underlying ambient space. Euler writes, "If the solid angle is formed from five plane angles..." [5, p. 6] Since Euler has chosen words for polyhedra that communicate a distinct relationship between the part and the whole, polyhedra are no longer identified with a spatial region. Polyhedra, and their parts, can now be considered apart from the underlying ambient space. Euler's conception of polyhedra can thus accommodate word choices that communicate operations on polyhedra that are nonsensical in Euclid's framework. In particular, Euler's polyhedra can be dissected into pieces. More generally, it is now feasible to discuss a polyhedron's solid angle apart from the ambient space and motion-based manner of construction, unlike on Euclid's conception. Indeed, Euler explicitly instructs the reader to consider a specific feature of polyedra apart from the underlying space and the way in which the polyhedron was formed - namely, convex polyhedra with planar faces: ${ }^{13}$

In Solid Geometry those bodies which are bounded on all sides by plane faces rightly merit first consideration, just as rectilinear figures do in Planar Geometry, or what is properly called Geometry. [5, p. 1]

After restricting his inquiry to planar-bounded solids, Euler states his purpose: to found solid geometry on sound first principles. Among Euler's first principles is his famous formula for polyhedra: "In every solid enclosed by plane faces the number of solid angles, along with the number of faces, exceeds the number of edges by two." [5, p. 2] Euler, despite being unable to prove his formula, maintains his "perception" of its truth for all solids [5, p. 2].

As Francese and Richeson note, Euler's formula in part arises from his coining a word for a polyhedron's edge, 'acies': "Remarkably, before Euler introduced it, no one had a name for an edge of a polyhedron." [6, p. 288] Echoing the remarks of Francese-Richeson, we note that this novel word choice foregrounded the features of a polyhedron that are related in Euler's formula. It was chiefly this new and (in Euler's time) non-standard word choice that spurred a conceptualization of polyhedron that led to a new and landmark development: "The importance of Euler's use of these three quantities should not be understated. Descartes came very close to stating Euler's polyhedral formula but was unable to do so because he had not singled out the edge as an important entity." [6, 288 $]^{14}$

Euler analogizes two dimensions and three dimensions in his proofs, leading him to propose the following method for studying three-dimensional figures:

In Geometry any rectilinear figure can be ultimately reduced to a triangle by successive division of angles. Likewise, given any solid enclosed by plane faces, I observed that the solid angles can be continuously divided so that, finally, a triangular pyramid remains. Since a triangular pyramid is the most simple figure among solids, I perceived that on the basis of its known properties one could generalize to the properties of all solids. [5, p. 2]

The idea of dividing - "dissecting" in Euler's vocabulary - a solid is introduced as an extension to three dimensions of the operation of dividing a planar figure [5, p. 3].

Note that dividing a polyhedron cannot be done so long as one conceives of polyhedra as Euclid did, in terms of a spatial region bounded by the trace of planar figures. For Euclid, one cannot divide the spatial region that constitutes the polyhedron, or separate the polyhedron's structure from its underlying and constitutive cordoned spatial region, without thereby annihilating the polyhedron. In contrast, Euler's conception of polyhedra, as communicated in his word choice, allows him to manipulate the solid and its pieces apart from the region of the ambient space that Euclid said constituted the polyhedron, thereby allowing Euler to study a polyhedron's structural features without regard for its manner of construction.

\footnotetext{
13 Francese and Richeson claim Euler assumed polyhedra to be convex [6, p. 289] [5, p. 1].

14 In contrast, Francese and Richeson note that there were old and familiar terms for face (hedra) and solid angle (angulus solidus) [6, 288].
} 
Euler rather describes solids as 'enclosed by plane faces' and not as constituted by being so-enclosed. To illustrate the distinction, consider cattle in a holding pen: the holding pen is the boundary, but it does not constitute the cattle in any sense of the word. For Euler, the polyhedron is conceived of as independent from its boundary, just as a cow is conceived of as independent from its enclosure. ${ }^{15}$ The phrase "cut a given solid angle from [a solid]..." lacks meaning unless the polyhedron has been conceived apart from its boundary and solid angles; similarly for the phrase "a part must be separated from the solid in such a way that solid angle $O$ is completely removed but everything else remains..." [5, p. 4] It is Euler's conception of polyhedra as apart from the underlying space that allows him to conceive of them with parts as distinct from the region of the ambient space that they occupy.

Euler does occasionally invoke motion language. In the piece we are discussing, Euler refers to a previous article ${ }^{16}$ in which he uses "drawing" in his proofs, arguing that since one can draw lines in two dimensions, one can draw planes in three:

If straight lines are drawn from any point within a solid to each solid angle, then the solid will be divided into as many pyramids as there are faces, in as much as each face will form the base of a pyramid, while their vertices meet at the point. [5, p. 3]

This process of drawing planes divides the polyhedron into multiple solid parts. Euler has a different method of decomposition in the article we are presently considering, a method "by which any solid is reduced by successive cutting of its solid angles to triangular pyramids." [5, p. 3] In the first article, Euler draws from a single interior point of a polyhedron to all of its vertices. In the article we treat in depth, Euler traces planes between solid angles [5, pp. 2-3]. Euler claims that, in either case, one arrives at triangular pyramids; the difference, Euler claims, consists merely in how one effects the decomposition. ${ }^{17}$

Euler describes the two-dimensional analogue of his three-dimensional method thusly:

This operation is similar to that by which any rectilinear figure is customarily reduced to a triangle by successive cuttings of its angles. For if we have a plane figure with sides $A B C D E F G A$, and if the triangle $C D E$ is cut from it by the line $C E$, the figure that remains is $A B C E F G A$, whose number of angles will be less by one. Now if, again, the triangle $C F E$ is cut by the line $C F$ the figure $A B C F G A$ will remain. If, from this, we next remove the triangle $B C F$ and then the triangle $B G F$, finally the triangle $A B G$ will remain [5, p. 3].

Euler's choice of the word 'cut' indicates a specific operation applied to the figure. This is reinforced by his repeating the phrase "successive cutting" to communicate his technique: "By successive cuttings of solid angles, I shall reduce all solids enclosed by plane faces, finally, to triangular pyramids." [5, p. 4] Euler describes this process as "applying the knife" to particular line segments. [5, p. 4] As indicated by his use of resulting pyramidal pieces to calculate the polyhedron's volume, Euler's cutting operation is a decomposition [5, p. 13].

But not all methods of decomposition are logically equivalent. If we interpret Euler's proof method as allowing the decomposition of the interior to be along arbitrary lines, then his proof contains a gap because his method of decomposing polyhedra into pyramids can generate non-convex solids from convex ones [6, p. 293]. But if Euler only permits planar decompositions of a polyhedron into pyramids, as his word choice of "cuts" and "applying the knife" strongly suggests, then Euler's proof does arguably preserve convexity as he claims; such a procedure applied to a convex solid always results in another convex solid. His word choice, that is, can close the gap in his proof discussed in [6, p. 292]. So depending on how closely we adhere to the conceptual framework which Euler encodes by his word choices ('cut', 'applying the knife'), we have either a gap-ridden proof or a gap-free proof.

So we have a conception of polyhedron, communicated by Euler's word choice, that is foreign to Euclid and allows for speaking of a polyhedron's structure as distinct from the underlying space and a motion-based means, or any other means, of constructing it. Not only that, but we have two readings of Euler's argument based on what his word choice conveys. If Euler allows any division of a polyhedron into pyramids, then his proof is faulty. If he only allows planar cuts that decompose a polyhedron into pyramids, then his proof is arguably correct. Word choice impacts his mathematics, enabling him to

\footnotetext{
15 For Euclid, it is rather that the object of study is the ambient space marked off by the holding pen.

16 Leonhard Euler, Elementa Doctrinae Solidorum" ("Elements of the Doctrine of Solids"), Novi Commentarii Academiae Scientiarum Petropolitanae 4: pp. 72-93, 1758.

17 As noted in [6, pp. 292-293] and [16, Chapter 3], Euler's claim is mistaken, at least on a natural reading.
} 
conduct studies of solid geometry that Euclid could not. Such consequences of word choice in mathematics indicates how word choice is critical in understanding and interpreting mathematics.

To summarize, word choices encode specific conceptualizations of the underlying objects of mathematical study, and thus distinct mathematical proofs and theorems arise from distinct word choices. Corresponding methods may even be rendered admissible or inadmissible by one's word choices.

Different interpretations of Euler's formula and Euler's proof, along with Euler's conceptual shifts, led to arguments over the legacy of Euler and his polyhedral formula. Some of these arguments revolve around word choice. We discuss these issues further in Section 3; presently, we conclude that Euler conceives of polyhedra as solids with component parts that may be removed or manipulated without destroying the solid, and that Euler's coining of the Latin word 'acies' for 'edge' partly spurred the discovery of his famous formula.

\section{Word Choice in Mathematical Texts}

We examine two book-length mathematical texts that illustrate vastly different developments of solid geometry and, correspondingly, exemplify vastly different modes of interaction between word choice and mathematical exposition. In the same text, one may adhere to a specific word choice, thereby employing a unifying conceptualization, or one may adroitly shift between word choices and the conceptualizations that they encode. In different contexts, with different aims or audiences, either approach may be hazardous. Some word choices may snugly suit the desired end of the exposition, or leave the audience bewildered and lost. So there is a balance to be struck between shifting word choices, on the one hand, and correspondingly shifting conceptualizations and adhering to a unifying word choice and developing mathematics within that one corresponding framework on the other. We study both ends on the spectrum to see how a text can advance a conceptualization of polyhedra via word choice.

\subsection{Coxeter's Regular Polytopes}

H. S. M. Coxeter's Regular Polytopes is a text in which there is dynamic shifting between word choices and, correspondingly, in conceptualizations of polyhedra; he shifts his word choice to adopt a conceptual orientation that eases the mathematical development at hand, fluidly moving between word choices that embody distinct conceptualizations.

Coxeter offers two basic definitions of 'polyhedron', one of which we term the "complex-definition" (here, 'complex' is a noun, in the sense of a conglomerate of interconnected parts) and the other we term the "figure definition." His figure definition appears in the preface: "A polytope is a geometrical figure bounded by portions of lines, planes, or hyperplanes; e.g., in... three [dimensions it is] a polyhedron." [2, p. vi] At first glance, this harks back to Euclid: "A solid is that which has length, breadth, and depth. An extremity of a solid is a surface." [4, Book 11, Definitions 1 and 2] Coxeter's figure definition of polyhedron defines the figure in terms of a bounded region of space; the boundary in turn is composed of previously defined component objects of lower dimension, namely lines and planes.

Coxeter's complex-definition appears in Chapter 1: "A polyhedron may be defined as a finite, connected set of plane polygons, such that every side of each polygon belongs also to just one other polygon, with the proviso that the polygons surrounding each vertex form a single circuit..." [2, p. 4] Coxeter's definitions of polyhedra shift between a bounded region of space and a set of oriented polygons. We will see that this makes a difference.

Coxeter invokes his complex-definition to define regular polyhedra: "A convex polyhedron is said to be regular if its faces are all regular and equal, and its vertices are all surrounded alike." [2, p. 5] Conceived as in the figure definition, as regions of space, Coxeter's polyhedra do not have vertices or other structural features that are separable from the underlying space. Conceived as in the complex-definition, as sets of oriented polygons, their structural features, such as their number of vertices, are brought to the fore. It is not accidental that Coxeter's definition of regular polyhedra draws on his complex-definition of polyhedra.

Coxeter's construction of the Platonic solids also relies on his complex-definition:

By placing two equal pyramids base to base, we obtain a dipyramid bounded by $2 p$ triangles... Similarly, by adjusting the altitude of an antiprism, we may take its $2 p$ lateral triangles to be 
equilateral... If $p=4$ or 5 , we can place pyramids on the two bases, making $4 p$ equilateral triangles altogether... There is no such simple way to construct the fifth Platonic solid. But if we fit six pentagons together so that one is entirely surrounded by the other five, making a kind of bowl, we observe that the free edges are the sides of a skew decagon. Two such bowls can then be fitted together, decagon to decagon, to form the dodecahedron... [2, pp. 5-6]

Coxeter's complex-definition leads his proof to proceed upon the arrangement of a polyhedron's polygons. Note that Coxeter's proof uses motion-language reminiscent of Euclid's.

Coxeter's treatment of symmetry also utilizes his complex-definition of polyhedra. "When we say that a figure is "symmetrical", we mean that there is a congruent transformation which leaves it unchanged as a whole, merely permuting the component elements." [2, p. 44] This definition requires that a polyhedron consist of structured components distinct from the ambient space. Coxeter also departs from motionlanguage here; a transformation is not a motion, but an action. Referring to points $\mathbf{A}_{n}$ which "may be regarded as the vertices of a generalized regular polygon," Coxeter observes "...there is a symmetry operation interchanging $\mathbf{A}_{n}$ and $\mathbf{A}_{-n}$ for all values of $n$ (simultaneously)." [2, p. 45] A simultaneous interchange of distinct objects is not a motion. Here the complex-definition is utilized.

Coxeter switches to his figure definition of polyhedron to discuss kaleidoscopes and reflections:

If the group [generated by reflections] is discrete, the whole set of planes effects a partition of space into a finite or infinite number of congruent convex regions, and the group is generated by reflections in the bounding planes of any one of the regions. Let these bounding planes or walls be denoted by $\mathbf{w}_{1}, \mathbf{w}_{2}, \ldots$ The dihedral angle between two adjacent walls, $\mathbf{w}_{i}$ and $\mathbf{w}_{j}$, is $\pi / p_{i j} \ldots$

[2, pp. 80-81]

In Coxeter's treatment of symmetry in general, his words describe actions on a polyhedron's component pieces. Here, in discussing reflections, Coxeter's object of inquiry is the underlying space and its regions; Coxeter uses words invoking a polyhedron's boundary. "In particular, two trihedra (or trihedral solid angles) are congruent if the three face-angles of one are equal to respective face-angles of the other." [ $[2$, p. 33] As Coxeter moves to name regions of reflection, he invokes the polyhedron's boundary, describing how one may "pass through" a region's wall [2, p. 81]. The aptness of this procedure depends on a conceptual shift from the complex-definition to the figure definition, which Coxeter readily undertakes.

Once this definition shift is made, motion language occurs. We mentioned "passing through"; we also have: "Whenever the path goes from one region into another and then immediately returns... when the path momentarily crosses an edge..." [2, pp. 80-81] Coxeter uses Euclidean language: we have "standing", "joined", and "inclined" in, respectively, $[2$, pp. $82,84,86] .^{18}$

Motion language also appears in Coxeter's treatment of star polyhedra:

The regular polyhedron $p$ is traced out by a moving point which continuously describes equal chords of a fixed circle and returns to its original position... The general regular polyhedron $p$ can be derived from the convex polyhedron $\mathrm{n}_{p}$ by either of two reciprocal processes: stellating and faceting. In the former process, we retain the positions of the sides of $\mathrm{n}_{p}$, and produce them at both ends, all to the same extent, until they meet to form new vertices. In the latter, we retain the vertices of $\mathrm{n}_{p}$ and insert a fresh set of sides, so that each new side subtends the same central angle... [2, pp. 94-95]

In addition to the use of motion-language, Coxeter opens his chapter on star polyhedra by invoking rotations to generate star polygons [2, p. 93]. He intermixes his two conceptions of polyhedra, discussing both the surface and an interior region in the same sentence: "By removing one zone from the triacontahedron, and bringing together the two remaining pieces of the surface, we obtain the rhombic icosahedron, which has a decidedly "oblate" appearance." [2, p. 29] Elsewhere, he writes, "[A] second rhombic dodecahedron... can be derived from the same rhombic icosahedron by removing one zone and bringing together the two remaining pieces of the surface." [2, p. 31] While describing the stellation of Platonic solids, he says, "In order to stellate a polyhedron, we have to extend its faces symmetrically until they again form a polyhedron." [2, p. 96] Here, Coxeter draws upon his polygonal notion of polyhedra; three pages later, he utilizes his figure definition: "Thus stellating involves the addition of solid pieces, while faceting involves the removal of solid pieces." [2, p. 99] Thus, Coxeter switches between definitions and their corresponding conceptions - and not fallaciously - throughout his text.

\footnotetext{
18 Euclid uses "passing through" and "joined" in [4, Book 13, Proposition 13]. Euclid uses both "standing" and "inclined" in, respectively, [4, Book 11, Definitions 5 and 7].
} 
We take Coxeter's dynamism in moving among conceptions as evidence of his geometric ability: Coxeter mastered multiple approaches to his objects of study. But we remark that Coxeter never explicitly mentions his shifts between conceptions, ${ }^{19}$ and only indirectly touches on this issue in the preface: "In fact, this book might have been subtitled "A sequel to Euclid's Elements"." [2, p. vi] He recognizes his geometric heritage from Euclid, leaving unmentioned his motion-language word choices - an inheritance from that same author.

\subsection{Grünbaum's Convex Polytopes}

Grünbaum, in contrast to Coxeter, adheres to one conception on polyhedra throughout his Convex Polytopes. As we will see, a polyhedron for Grünbaum is conceived with the language of sets: it is a set of points in the Euclidean space $\mathbb{R}^{d}$ with certain properties.

Grünbaum begins his book by limiting the scope of the inquiry: "With few exceptions, we shall be concerned with convexity in $R^{d}$, the $d$-dimensional real Euclidean space." [7, p. 1] Grünbaum develops a conception of polyhedra as sets of $d$-tuples in $\mathbb{R}^{d}$. He defines geometric entities as sets of such points. ${ }^{20}$ For example, Grünbaum writes, "A hyperplane $H$ is a set which may be defined as $H=\left\{x \in R^{d} \mid\langle x, y\rangle=\alpha\right\}$ for a suitable $y \in R^{d}, y \neq 0$, and $\alpha . "$ [7, p. 2] Similarly, Grünbaum writes, "Let $K$ be a convex subset of $R^{d}$. A set $F \subset K$ is a face of $K$ if either $F=\varnothing$ or $F=K$, or if there exists a supporting hyperplane $H$ of $K$ such that $F=K \cap H . "$ [7, p. 17] His adoption of the convention dimension $(\varnothing)=-1$ shows that Grünbaum aims to explicitly channel his conception of polytopes as sets of $d$-tuples in his technical development and even his notation $\left[7\right.$, p. 3]. ${ }^{21}$ Another example of his strict adherence to a single conception of polyhedra involves his definition of 'section': "A section of a polytope $P$ is the [set] intersection of $P$ with some flat..." [7, p. 71]

Furthermore, Grünbaum couches spatial relationships in set-language: "A set $A \subset X$ is bounded if there exists $\delta>0$ and $x \in X$ such that $\rho(a, x)<\delta$ for all $a \in A$." $[7, \text { p. } 5]^{22}$ Grünbaum also defines convexity in the language that utilizes operations on sets:

A set $K \subset R^{d}$ is convex if and only if for each pair of distinct points $a, b \in K$ the closed segment with endpoints $a$ and $b$ is contained in $K$. [So] $K$ is convex if its intersection with every straight line is either empty, or a connected set. [7, p. 8]

Grünbaum is indifferent as to whether we rely on subset or intersection to ground convexity, but either way, we consider convex figures as sets of $d$-tuples. Grünbaum also conceptualizes the relationships 'beyond' and 'beneath' in set-language [7, p. 78].

Similarly, Grünbaum defines historically spatial operations in set-language. For Euclid, motion language grounds words like 'cut'; for Grünbaum, set-language explains the meaning of 'cut':

Let $A$ be a subset of $R^{d}$. We shall say that a hyperplane

$$
H=\left\{x \in R^{d} \mid\langle x, u\rangle=\alpha\right\}
$$

cuts $A$ provided both open halfspaces determined by $H$ contain points of $A$. In other words, $H$ cuts $A$ provided there exist $x_{1}, x_{2} \in A$ such that $\left\langle x_{1}, u\right\rangle<\alpha$ and $\left\langle x_{2}, u\right\rangle>\alpha$. [7, p. 10]

So Grünbaum, rather than conceiving of a 'cut' as a spatial action, conceived of a 'cut' as a relationship between sets.

Grünbaum proceeds with a similarly set-theoretic conception in constructing objects of study: "To 'construct' means to draw appropriate lines in the plane, i.e. to specify a suitable configuration." [7, p. 94] Here Grünbaum unpacks 'to draw' as specifying a configuration of points in a coordinate space. And Grünbaum defines a configuration as "a finite set of points and lines in a projective plane, with

\footnotetext{
19 This is different from generalizing a definition within one conception, as here: "the definition of polyhedron can be generalized by allowing non-adjacent faces to intersect..." [2, p. 96] We remark that it is important to explicitly recognize when conceptual shifts occur so as to facilitate understanding. If, say, a student-reader might miss such conceptual shifts, explicitly marking them in one's treatment seems warranted.

20 Part of our point is that one can approach the same entities from other vantage points; labeling polytopes as 'geometric' may bias one perspective over other possibilities, as Grünbaum's text shows.

21 Instead of, say, regions of space or polygonal arrangements. Coxeter does not adopt this convention.

22 The departure from Euclid here is striking; containment is no longer conveyed by a spatial metaphor.
} 
prescribed incidence relations." [7, p. 93] Similarly, terms like 'separates' and 'supports' each receive similar set-theoretic casting [7, p. 10], as do both 'pasting' [7, p. 59] and 'pulling' [7, p. 80]. Grünbaum meticulously keeps to his set-language in presenting his geometry.

Grünbaum's theorems and proofs also conceptualize historically geometric notions in the language of sets. Consider the following two theorems and some excerpts from the proof of the first theorem:

The following results are of fundamental importance.

1. If $A$ and $A^{\prime}$ are convex subsets of $R^{d}$ such that $A^{\prime}$ is bounded and $\operatorname{cl} A \cap \operatorname{cl} A^{\prime}=\varnothing$, then $A$ and $A^{\prime}$ may be strictly separated by a hyperplane.

2. If $A$ and $A^{\prime}$ are convex subsets of $R^{d}$ such that $\operatorname{aff}\left(A \cup A^{\prime}\right)=R^{d}$ then $A$ and $A^{\prime}$ may be separated by a hyperplane if and only if

$$
\text { relint } A \cap \operatorname{relint} A^{\prime}=\varnothing \text {. }
$$

PROOF OF THEOREM 1 Since the distance between two sets... Therefore $\bigcap_{\epsilon>0}\left(A^{\prime} \cap B(\epsilon)\right)=$ $A^{\prime} \cap B(0) \neq 0$. In other words, there exists a point $y=y(x) \in A^{\prime}$ such that $\delta\left(x, A^{\prime}\right)=\rho(x, y(x)) \ldots$ Each point of the first interval, sufficiently near to $x_{0}$, is at a distance less than $\delta$ from $y\left(x_{0}\right)$ and thus can not belong to $A$; similarly, each point of the second interval... Since both $A$ and $A^{\prime}$ are convex, it follows that $z$ belongs to neither of them... [7, p. 11]

Rather than talking about spatial position or using motion language, Grünbaum uses set language, ${ }^{23}$ reasoning about a historically spatial concept (convexity) using the language of a set-theoretic notion (membership). Another example of conceptualizing in the language of sets what has historically been a motion occurs in [7, pp. 83-84], a typical modern application of the notion of convergence. Set-theoretic language influences Grünbaum's vocabulary and proof methods.

We conclude with a look at Grünbaum's treatment of polytopes and Euler's formula. Grünbaum defines, "A compact convex set $K \subset R^{d}$ is a polytope provided ext $K$ is a finite set." [7, p. 31] This is a set-theoretic conceptualization of the notion of a polytope. He even uses set-language to motivate Euler's formula: "What $d$-tuples of numbers can occur as the numbers of vertices, edges, $\ldots,(d-1)$-faces of convex $d$-polytopes?" [7, p. 130] He answers:

We formulate the result known as Euler's theorem:

1. The affine hull of the $f$-vectors of all members of the family $\mathscr{P}^{d}$ of all d-polytopes is given by

$$
\operatorname{aff} f\left(\mathscr{P}^{d}\right)=\left\{f=\left(f_{0}, \ldots, f_{d-1}\right) \mid \sum_{i=0}^{d}(-1)^{i} f_{i}=1-(-1)^{d}\right\} .
$$

The relation $\sum_{i=0}^{d}(-1)^{i} f_{i}=1-(-1)^{d}$, which by the theorem holds for every $d$-polytope $P$, is known as Euler's equation. [7, p. 131]

Grünbaum's conceptualization of $V+F=E+2$ ties Euler's formula to set-talk. Grünbaum is well-aware of this! He remarks:

Some remarks of a methodological nature seem indicated in view of the proofs given in the present Section [Polytopes]. It is hoped that readers who worked their way through the proofs are by now ready to accept the validity of the results proved. The author doubts, however, that the above formal proofs give a good idea of why the proofs work. In a subject as elementary and intuitively comprehensible as the theory of polytopes, it seems a pity to obscure the simple idea of a proof by the - almost necessarily - involved and complicated notation and symbolism... In this context, as in many other cases, the idea of the proof becomes clearly comprehensible with the help of a graphic presentation of the two- or three-dimensional case... [7, p. 33]

\footnotetext{
23 A non-exhaustive list of examples include the definition of convex hull and Carathéodory's Theorem on [7, p. 14-15], poonem (Hebrew for 'face') [7, p. 20], unboundedness [7, p. 23], the notion of a polytope's dual [7, p. 46], combinatorial scheme [7, p. 90], and the Euler characteristic of a proper face [7, p. 138].
} 
Grünbaum acknowledges that the reader may have difficulty visualizing the proven facts due to his "notation and symbolism," which we have noted is heavy with set-theoretic word choices. While adherence to a particular framework in approaching an object of study can preserve purity of perspective, it can also result in confusion at certain steps of the treatment, at least for those readers who conceive of polyhedra through different, e.g. spatial, word choices. The words a mathematical writer chooses bear significantly on his or her success in communicating to the reader a solid connection between the underlying intuitions and the objects of study.

\subsection{Word Choices as Codifying Conceptualizations}

David Reed's Figures of Thought studies mathematical texts "as texts", i.e. as encoding a conceptual stance towards their objects [15, p. x]. He writes:

Although all mathematics is set forth in texts which argue, explicitly or implicitly, for their own version of 'doing mathematics'... the general view remains that mathematical facts and mathematical subject matters exist somehow independently of the texts in which they are expressed. $[15$, p. xi]

Reed aims to refute this "general view". Using Euclid as his case study, Reed studies Euclid's approach to mathematics and argues that critiques of Euclid's 'imprecision' or 'gap-ridden' proofs have ignored the conceptual setting of Euclid's arguments [15, p. xii-xiii].

Reed advances the claim that Euclid's work presents a systematized whole, one that moves from a study of parts in Books I-X to wholes in Book XI-XIII [15, p. 164]. On his account, Euclid's Elements has been anachronistically misrepresented as containing bad proofs and other flaws by the axiom-driven mathematicians of the modern era, who had goals and standards for rigor rather different from Euclid's own [15, p. 162-163]. They pursued a form of completeness in geometry that Euclid did not espouse:

...from Euclid's point of view... [t]he study of plane geometry does not constitute a whole and cannot achieve completion; the study of solid figures in which plane figures function as boundaries and parts is a study of wholes and arrives at a natural conclusion. [15, p. 145]

Reed argues that Euclid's work, from start to finish, follows a careful plan and explores the limits of definability, and that contemporary authors ignore the philosophical elements underlying Euclid's reasoning [15, p. 162].

We mention Reed's thesis because he highlights a phenomenon of mathematical practice: the conceptualization(s) of a mathematical subject-matter embodied by an entire text. We here focus on the phenomenon of word choice as encoding a conceptualization of the mathematical objects of study. This phenomenon resurfaces in a recent dispute over the appropriate word choice for and conceptualization of polyhedra, as we will see in Section 4.

Reed also discusses the vital role of conceptualization. His remarks equally apply to whole texts or individual word choices:

Mathematical understanding is achieved by finding appropriate mathematical terms in which problems can be formulated and theorems enunciated. It might be said that the mathematics in a mathematical text is precisely that in it which can be translated into other (mathematical) terms.

The text and its argument is what cannot be translated or so transformed. [15, p. 161]

As with translating languages, moving from one conceptualization to another may result in some loss of information or rigor, or gains in the same. As we have seen in the above discussion, word choice is a factor in conceptualizing an object of mathematical study.

Grübaum is alert to this fact. He notes that a text encodes a conceptualization, or conceptualizations, of polyhedra: he writes, “...'polyhedron' can mean either a solid... or a surface..." [8, p. 461] Grünbaum brings this up in discussing past attempts at complete classifications of regular polyhedra and persistent counterexamples to them. He argues:

...all the results mentioned are completely valid; what changed is the meaning in which the word "polyhedron" is used. As long as different people interpret the concept in different ways there is always the possibility that results true under one interpretation are false with other understandings... even slight variations in the definitions of concepts often entail significant changes in results. $[8$, p. 462$]$ 
A similar sentiment occurs in Lakatos' Proofs and Refutations; informal proofs that we find acceptable are indexed to a conceptualization [12, p. 56]. We can switch conceptual frameworks, changing, say, the accompanying word choices, and this can invalidate a given result. Grünbaum observes:

If [Kepler's star polyhedra] are interpreted as solids, their boundary is a topological sphere bounded by sixty triangles in each case. Under this interpretation, neither of the two polyhedra is regular. However, they are regular if they are interpreted the way Kepler intended, as being constituted by 12 pentagrams ("star pentagons") each. [9, p. 451]

Moreover, by their effects on the reader or researchers' conceptualization of the object of study, word choice can foreclose possibilities and discoveries to the mathematician: "But the impossibility of Steinitz having used this formulation is obvious from the fact that in 1916 nobody used terms such as ' 3 -connected graph'." [9, p. 448] Switching to a different conceptualization can clarify a proof or bring out a feature of the object being studied, but such switches in conceptualization can also obstruct a proof:

However, the ideas of Steinitz's original proofs can be reworked in a graph-theoretic setting, gaining in clarity and simplicity. [9, p. 447]

Eberhard's proof is long and messy, since he has to accommodate the operations he performs on the convex polyhedra. A simpler proof, utilizing graphs and Steinitz's theorem, appears in $[18$, Section 13.3]. [9, p. 450]

Researchers and teachers can choose from conceptual frameworks available: one may select more than one framework, but at least one framework must be chosen. This selection may result in varying degrees of successful communication and, importantly, this selection impacts the mathematics developed - proofs, techniques, and theorems. Success depends partly on the target audience.

On the one hand, the dynamism of Coxeter may be easier to follow with a high degree of exposure to the subject. On the other hand, the new student may find the interconnections bridged in language compelling, which could inspire greater interest in the subject. Again, on the one hand, a stricter presentation like that of Grünbaum may provide greater intelligibility of the argument in the sense that each step can be followed more easily. On the other hand, the global perspective may be lost in the process.

The 'right' approach(es), if any, can only be decided on a case-by-case basis: the preferred conceptual background, and so the preferred choice of words, may change with the author's conceptualization of the subject-matter, the intended goal(s), and the audience(s). Facilitating mathematical communication and understanding manifestly requires sensitivity to the conceptualization(s) encoded in one's word choices.

\section{Euler's Formula}

We next discuss a recent dispute as to what Euler meant by his formula for polyhedra. To introduce this dispute, we first revisit the language with which Euler conveys his formula in "Proof of Some Notable Properties", and then we discuss Imre Lakatos' Proofs and Refutations, which treats the formula in detail. We will then consider 'conflicting' interpretations of Euler's formula offered by two pairs of modern authors. We draw attention to the fact that they are partly disputing word choice for polyhedra.

Euler makes his formula part of the basis for his solid geometry:

...the general properties of solids... depend on one property [Euler's formula] in such a way that if it were possible to prove this property, then all of the first principles of Solid Geometry which I have proposed would be equally as firm as the first principles of Geometry. [5, p. 1]

Recall that Euler's formula, in his (translated) words, is this: "In every solid enclosed by plane faces the number of solid angles, along with the number of faces, exceeds the number of edges by two." [5, p. 2] In notation, this reads $V+F=E+2$.

Then there is the matter of proving it. Euler describes his proof method as follows:

I arrived at proofs similar to the one customarily used for the analogous proposition from Geometry regarding the sum of the angles of any rectilinear figure. In Geometry any rectilinear figure can be ultimately reduced to a triangle by a successive division of angles. Likewise, given any solid enclosed by plane faces, I observed that the solid angles can be continuously divided so that finally, a triangular pyramid remains... Here I will explain a... method by which any solid is reduced by successive cutting of its solid angles to triangular pyramids. [5, pp. 2-3] 
Euler describes his proof as shearing off solid angles from the solid one at a time and bases his argument on the equation $V+F=E+2$ holding after each removal [5, p. 12]. From these excerpts, we know Euler's proof requires that a polyhedron, as a region of space bounded by planes, be amenable to decomposition into components of space, e.g., into pyramids. If the solid is conceived as constituted by its boundary, as in Euclid, then this process is incoherent.

Euler's language does not disambiguate his notion of 'counting' vertices, faces, and edges; the counting procedure occurs vicariously via triangular pyramids. His statement only requires that the number of vertices, edges, and faces satisfy his formula, but how one determines the number of vertices, edges and faces remains open. Not surprisingly, given this ambiguity, the meaning of Euler's formula has long been a matter of dispute, as Lakatos' book exploring the history of Euler's formula shows.

Lakatos' Proofs and Refutations: The Logic of Mathematical Discovery explores relationships between theorem, proof, and counterexample using Euler's formula as the case study. He tracks attempts of a hypothetical class of students to prove Euler's formula definitively, i.e., without gaps appearing or counterexamples. Lakatos' fictional students explore the content, truth, applicability, and meaning of Euler's theorem: they catalog, in their offered guesses and counterexamples, historical instances of mathematicians undergoing the same process.

A fictional teacher leads the students through speculation as to the relationship between the number of vertices, edges, and faces of a polyhedron, asking them to consider whether some relation, analogous to $V=E$ for polygons, holds for polyhedra [12, p. 6]. Most of this discussion paraphrases quotes, proofs, theorems, and counterexamples from the historical development of the mathematics of polyhedra. ${ }^{24}$ The teacher offers a proof lifted from Cauchy [12, pp. 7-8], which the students subsequently consider and refute with a counterexample [12, p. 10]. The students then engage in offering improved definitions, including those offered by Legendre, Jonquières, Möbius, and Baltzer, and new counterexamples, some of which may be found in the work of Lhuilier, Hessel, and Kepler. ${ }^{25}$

Lakatos' fictional setting distorts little of the troubled history of Euler's formula: counterexamples - solids satisfying a given definition of a polyhedron for which $V+F=E+2$ does not hold - have repeatedly been offered in response to proposed proofs of the result; in turn, these counterexamples (or 'monsters') have been 'barred' by improvements to the proof or the incorporation of lemmas; labeled 'exceptions' and so excluded from the theorem's domain of application; ignored altogether; or admitted into a revised 'Euler's formula' or definition of 'polyhedron' that masks changes to the proof. Save for the fictional pupils and dialogue, the result is an accurate depiction of the history of Euler's formula.

Lakatos' imaginary pupil Alpha describes this procedure: “...the identity of the linguistic expressions of the naive conjecture and the monster-barring theorem hides, behind surreptitious changes in the meaning of the terms, an essential improvement." [12, p. 41] The phenomenon Alpha is alluding to is how changes in word choice invite reconceptualization of objects of mathematical study and thus mathematical changes.

We next consider a modern instance of this phenomenon: In relatively recent issues of the American Mathematical Monthly, two pairs of authors debated the meaning of Euler's formula. Both pairs use the term 'polyhedron', but this hides their differing interpretations of Euler's theorems. One pair emphasizes Euler's word "counting"; the other pair emphasizes Euler's word "division" (of space). We shall see that these word choices demarcate distinct conceptualizations of polyhedra.

\subsection{Grünbaum's and Shephard's "A New Look at Euler's Theorem for Polyhedra"}

Branko Grünbaum and Geoffrey Colin Shephard aim to incorporate all counterexamples to Euler's formula under one conceptual apparatus, "polyhedral sets." Grünbaum and Shephard intend polyhedral sets to rigorously capture the act of counting vertices, edges, and faces of a polyhedron [11, Response from Grünbaum and Shephard, p. 962]. They hold that any polyhedron will fall under the framework of polyhedral sets: "polyhedral sets... generalize familiar polyhedra; they may be closed, open, or neither, connected or not, bounded or not, and their parts may have different dimensions." [10, p. 110]

\footnotetext{
24 Much of this historical background receives a masterful treatment in Peter R. Cromwell's Polyhedra; we recommend [3, pp. 181-218], especially [3, pp. 198-210].

25 [12, p. Legendre, 14, Footnote 1], [12, p. Jonquières, 14, Footnote 2], [12, p. Möbius, 15, Footnote 2], [12, p. Baltzer, 16, Footnote 1], [12, p. Lhuilier, 13], [12, p. Hessel, 15], and [12, p. Kepler, 16, Footnote 2].
} 
For Grünbaum and Shephard, the domain of objects caught under the umbrella word 'polyhedra' includes any geometric realization of a set of vertices and edges, so long as these can be counted by the methods they describe. The word "counting" sets the limits of inquiry [11, Response, p. 962]. What justifies their use of 'polyhedron' to refer to such a wide variety of objects? They argue that their view is "simple and elementary," relating "an integer $\chi(P)$ to the geometrical features of $P$," where $P$ is a polyhedral set [10, pp. 110-111]. Grünbaum and Shephard intend $\chi(P)$ to be a generalization of Euler's formula.

Grübaum and Shephard define a "closed convex polyhedron" as "the intersection of a finite number of closed half-spaces." [10, p. 114] They allude to the inclusion of traditional examples of polyhedron while stressing their notion's broader applicability:

Familiar examples of closed convex polyhedra are (closed) cubes, (closed) squares, (closed) line segments, and single points... But our definition includes also unbounded convex polyhedra... their edges can be rays (halflines) or straightlines, and faces and cells can be unbounded as well. [10, p. 114]

We arrive at Grünbaum and Shephard's notion of polyhedral set:

If a set $P$ is the union of members a finite family $\mathcal{C}=\left\{C_{1}, C_{2}, \ldots, C_{n}\right\}$ of pairwise disjoint sets $\left\{C_{1}, C_{2}, \ldots, C_{n}\right\}$, we say that $\mathcal{C}$ is a dissection of $P$ and we write $P=\dot{\cup} \mathcal{C}$, where the dot in the union symbol indicates that the sets $C_{i}$ are pairwise disjoint. If $\mathrm{P}$ is a set that admits a dissection $\mathcal{C}$ all elements of which are relatively open convex polyhedra we shall say that $P$ is a polyhedral set, and express this by writing

$$
P=\dot{U}_{c} \mathcal{C}
$$

where the subscript $c$ is to remind us that each element of $\mathcal{C}$ is a relatively open convex polyhedron.

Grübaum and Shephard remark once again that their definition is quite general:

It will be observed that the definition is very general in that it does not require $P$ to be open or closed, connected, simply connected or even homogenous in the sense that neighborhoods of all points of $P$ are of the same dimension. [10, p. 116]

By only demanding that a polyhedron's pieces be separable and requiring nothing of the polyhedron's underlying space, e.g., that all pieces have the same dimension, Grünbaum and Shephard depart from the language of space of Euler's original proof, the language that enables a polyhedron to be cut into pyramidal pieces.

The upshot of their departure from Euler's language of space is new examples for consideration. "All the sets shown in Figures 1 to 9 are polyhedral sets..." [10, p. 116] Ever-further generalization drives Grünbaum and Shephard's extension of Euler's formula:

Thus the definition of $\chi(P)$ given here may be regarded as an extension of the traditional approach to sets which need not be closed. On the other hand, our definition is restricted to polyhedral sets, hence not applicable to non-polyhedral sets even if they are not convex. [10, p. 117]

Their definition of $\chi(P)$ for a polyhedral set $P$ is as follows [10, p. 117]:

1. $\chi(\emptyset)=0$

2. If $P$ is a relatively open convex polyhedron of dimension $d$, then $\chi(P)=(-1)^{d}$.

3. If $P=\dot{U}_{c} \mathcal{C}$ is a relatively open convex dissection of $P$, then $\chi(P)=\sum_{C \in \mathcal{C}} \chi(C)$.

How does $\chi(P)$ capture $V+F=E+2$ ? Grünbaum and Shephard introduce four $k$-scaffolds of a polyhedral set $P$. These sets are defined as follows [10, p. 118]:
1. $\operatorname{scaf}_{3}(P)=\operatorname{int}(P)$
2. $\operatorname{scaf}_{2}(P)=\operatorname{int}\left(P \backslash \operatorname{scaf}_{3}(P)\right)$
3. $\operatorname{scaf}_{1}(P)=\operatorname{int}\left(\left(P \backslash \operatorname{scaf}_{3}(P)\right) \backslash \operatorname{scaf}_{2}(P)\right)$
(interior points)
4. $\operatorname{scaf}_{0}(P)=\operatorname{int}\left(\left(\left(P \backslash \operatorname{scaf}_{3}(P)\right) \backslash \operatorname{scaf}_{2}(P)\right) \backslash \operatorname{scaf}_{1}(P)\right) \quad$ (vertices) 
Grünbaum and Shephard do not care, for example, what dimension of space or region of space a polyhedron's parts occupy; their scaffolding will capture vertices, edges, and faces because the structure of the parts, how one "divides" a polyhedron, does not impact the tabulation of a polyhedron's components: "the definition of $\operatorname{scaf}_{k}(P)$ is independent of the relatively open convex dissection of $P$ into polyhedral sets." [10, p. 118]

We have here an interpretation of the Euler characteristic such that the polyhedron's structure can be as 'bizarre' as one pleases. A polyhedron's structure is given and not adjusted to fit the Euler characteristic; rather, the Euler characteristic accommodates whatever the polyhedron's structure may be. We see a very different perspective in the next section.

We need not speculate as to Grübaum and Shephard's purpose, as they explicitly state their dissatisfaction with the history of Euler's theorem: "At least two books have been dedicated to the early history and attempts at clarification of Euler's theorem." [10, p. 122] They spend about six pages of the article detailing past confusions, and then give the following diagnosis:

All the discussions of [Euler's theorem] were dogged by two difficulties... On the one hand, no precise definitions were given for the polyhedra under consideration or for their faces, edges, and vertices. It was more or less generally assumed that one is dealing with solids and considering features on their surfaces - but how to determine faces (or edges) was illustrated by examples rather than defined by unambiguous rules... On the other hand, in its original formulation and in the minds of many early workers, it was a hallmark of Euler's theorem that it involved only the numbers of vertices, edges, and faces - without any consideration of the nature of the faces. [10, p. 123]

Grünbaum and Shephard claim to clarify Euler's theorem and preserve Euler's insight. They believe they have provided definitive rules for counting edges, faces, and vertices which are guided by Euler's language, especially his choice of the word 'counting'. On the other hand, Grünbaum and Shephard find that focusing on space obscures the idea of Euler's formula:

Starting with Poincaré... these concepts acquired precise meaning for simplicial complexes and more general objects... However, this development led to a loss of the connection to the origins of Euler's theorem as a relation involving the vertices, edges and faces of a polyhedral objects. [10, p. 125]

They similarly discount Betti numbers as a precise interpretation of Euler's original insight. [10, p. 123] This evaluation is contrary to Hilton and Pedersen's conceptualization of polyhedra, which we consider in the next section.

\subsection{Hilton's and Pedersen's "Euler's Theorem for Polyhedra: A Topologist and Geometer Respond"}

Peter Hilton and Jean Pedersen approach polyhedra through the perspective of underlying space. They dispute Grünbaum's and Shephard's version of the history of Euler's theorem:

[Grünbaum's and Shephard's] paper appears, in contrast to its obvious positive contribution, to both neglect and distort the contribution which algebraic and combinatorial topologists have made to the development of the Euler characteristic. We claim that the 'problem' referred to on page 110 of $[\mathbf{1}] \ldots$ is no real problem at all, and that topologists have completely and satisfactorily formalized the Euler characteristic... Moreover, we categorically deny that 'this development led to a loss of the connection to the origins of Euler's theorem as a relation involving the vertices, edges and faces of a polyhedral object.' [11, p. 959]

Hilton and Pedersen believe that topologists solved all conceptual difficulties relating to the Euler characteristic and that no controversy obtains over the present state of the theorems. Note that they do not contest the mathematical merit of Grünbaum and Shephard's article:

...resemblance of their Theorem 4 to the classical theorem of Euler might seem at first to owe more to a judicious choice of notation than to a conceptual closeness; however, careful inspection of their definition of a $k$-scaffold and contemplation of their examples show that their notation may, indeed, be justified. [11, p. 959] 
Hilton and Pedersen do not dispute Grünbaum and Shephard's mathematical innovation, yet they argue that Grünbaum and Shephard's rendition of the history of Euler's formula wrongly identifies the content of Euler's theorem as concerned with the act of counting; Hilton and Pedersen believe the topological development of Euler's formula remains true to Euler's development of his formula as a description of spatial properties:

We regard the theorem (referred to somewhat disparagingly in [1]) that the Euler-Poincaré characteristic equals the alternating sum of Betti numbers as fundamental. It not only explains Euler's original result (since orientable closed surfaces bounding convex regions are homeomorphic to the sphere), but establishes the homotopy invariance (not merely topological invariance) of $\chi$. Of course, it also explains formula (2) of [1], that is

$$
v-e+f=2-2 g,
$$

for an orientable closed surface of genus $g$, since the Betti numbers of such a surface are given by $p_{0}=1, p_{1}=2 g, p_{2}=1$. Thus, formula (2) is scarcely a stride in extending Euler's Theorem and so should not be seen as 'dealing with a discrepancy'. [11, p. 960]

Hilton and Pedersen think that the topological development of Euler's theorem exposes Euler's true insight, and that Euler's theorem gives an invariant between surfaces, i.e., that solids with different Euler characteristics are (topologically-speaking) different. The notion of counting is entirely absent from their discussion; Hilton and Pedersen emphasize Euler's language of dividing or decomposing a space over his language of counting.

Hilton and Pedersen claim that formula (2), i.e., $v-e+f=2-2 g$, is a case of Euler's formula rather than an extension, and argue against Grünbaum and Shephard's expansion of the term 'polyhedron':

Indeed, one might legitimately comment that it is unreasonable to expect that one can count the number of holes by counting subsets which themselves are allowed to have holes! Moreover, if one allows strange (2-dimensional) faces... then why not regard the entire surface as a face and thus obtain $v-e+f=1$ for any surface! Finally, we remark that it should never happen that scratching a few extra lines on a surface... should alter a really significant invariant quantity.

...Nor should Figure 2 of $[\mathbf{1}]$ create a problem... Certainly $\chi(P)=1$ for this polyhedron, so formula

(2) does not apply. But $P$ is not a closed orientable surface, so how could formula (2) apply? [11, p. 960]

Hilton and Pedersen deny Grünbaum and Shephard's application of Euler's theorem (to them, a fact about space) to some objects with bizarre (to Hilton and Pedersen) underlying spaces, such as topologically nontrivial two-dimensional faces [11, p. 960]. Their conceptualization of polyhedra as intimately bound to the underlying (topological) space - encoded in their spatial-language word choices - leads them to such conclusions. They see, e.g., an "error in permitting Figure 1(b) as a legitimate dissection of the torus..." [11, p. 960]

Hilton and Pedersen specify what they consider the appropriate domain of inquiry ${ }^{26}$ corresponding to the term 'polyhedron' by offering a characterization of the objects of study:

We claim that a polyhedron is made of cells and a closed (n)-cell is the homeomorph of the $n$-dimensional ball $x_{1}^{2}+x_{2}^{2}+\cdots+x_{n}^{2} \leq 1$ in $\mathbb{R}^{n}$. In particular, a two dimensional face must be a polygonal region [11, p. 959-960].

In other words, the substructure of a surface does not matter; a polyhedron's regions must merely be amenable to deformation into cells for the solid to count as a polyhedron.

Grübaum and Shephard, on the other hand, deny in their response the 'spatial divisibility' limits Hilton and Pedersen would apply to the polyhedra:

In particular, we reject the implication that the geometrical approach we propose is redundant. The purpose of our paper... is to show how the "traditional" approach of counting vertices, edges, and faces, can, with care and appropriate modifications, lead to results which are valid and far-reaching. In particular, there is no need, as topologists generally do, to restrict attention to bounded polyhedra which can be expressed as finite unions of topological balls all of which are 26 Lakatos calls this the "domain of proof," but here we use the term in a slightly broader sense that corresponds to an
area of mathematical inquiry rather than a single proof from such an area [12, p. 64]. 
open or all of which are closed... it seems unreasonable to us to reject a polyhedron... by stating dogmatically that every "2-dimensional face must be a polygonal region" and then to define a polygonal region in such a way as to eliminate multiply-connected regions. Euler himself, if he were alive today, would, we believe, feel far more comfortable with our approach to his theorem than that advocated in the "Response"... Our purpose... was to show that a simple geometrical treatment of a classical topic can lead to results that are more general, and more intelligible to the majority of mathematicians, than the recondite and much more abstract topological approach.

[11, Response, p. 962]

Grünbaum and Shephard make it explicit: their conceptualization of Euler's formula ties it to counting. They find Hilton's and Pedersen's spatial restrictions unappealingly ad hoc. Again, the mathematical results are not issue; the issue is what one means by 'polyhedron', and what Euler meant 240 years ago in formulating his formula. The same word, 'polyhedron', conveys two different concepts based on Euler's choice of two other words, 'sum' and 'divides', to describe his fundamental result. We see this by noticing how one pair of authors uses counting language while the other uses spatial language. (Interestingly, each side of the dispute traces their word choices and conceptualization back to Euler's words explicitly without ever citing Euler's words.)

This argument between the two pairs of authors over Euler's formula turns chiefly on their divergent word choices for their respective conceptualizations of polyhedra. The different conceptualizations lead each pair of authors to use distinct word choices - 'count' and 'spatial divisibility' - to direct their mathematical discussion of Euler's formula. The pairs of authors disagree about the set of objects to which the word 'polyhedron' rightfully applies and the meaning of Euler's formula: is it the division of space or the act of counting that Euler emphasized? Euler himself uses both the language of counting and the language of dividing space, and his text does not seemingly decide which choice of words is the right rendering of his meaning some 240 years ago. The result of these divergent word choices is a conceptual dispute between mathematicians over the appropriateness, motivation, and meaning of ostensible developments of Euler's formula and its modern interpretation. ${ }^{27}$

For Hilton and Pedersen, a (three-dimensional) polyhedron must be decomposable into closed balls homeomorphic to a sphere and the faces must be homeomorphic to a circle; moreover, certain alterations to the polyhedron must not change the Euler characteristic of a polyhedron. For Grünbaum and Shephard, the polyhedron need not be decomposable into 'nice' pieces and Euler's characteristic does not track topological invariance; Euler's formula applies to any polyhedron if one employs precise notions of vertex, edge, and face.

The crucial point is that the argument revolves around the range of objects to which the concept expressed by the word 'polyhedron' applies. This disagreement is manifested in the divergent word choices for the objects of study. Whether we use the language of counting or of dividing space alters which conceptualization of Euler's formula, and of polyhedra, we study. The mathematical realization of the concept, and thus the direction of one's inquiry, will be correspondingly different. It will not thereby be 'wrong'.

\section{Conclusions}

As we stated in Section 1, our case study of 'polyhedra' evidences the influence of word choice on mathematical practice. This relates to other meta-mathematical issues relevant to mathematical practice. For example, Lakatos' work highlights the importance of guesswork and counterexamples in producing theorems with rigorous proofs $[12, \mathrm{pp} .73-74]$. He also makes insightful remarks about language influencing mathematics.

Pi: ...I certainly wouldn't call a whale a fish, a radio a noisy box... and I am not upset when a physicist refers to glass as a liquid. Progress indeed replaces naive classification by theoretical classification, that is, by theory-generated (proof-generated, or if you like, explanation-generated)

\footnotetext{
27 One might prefer to say that the conceptual disagreement leads to divergent word choice. Here we stake no claim as to the priority of explanation - though there is no doubt about the close tie between word choice and conceptualization since our purpose is to examine the vital phenomenon of word choice in mathematical practice, particularly as encoding a conceptualization of mathematical subject-matter.
} 
classification... Naive conjectures and naive concepts are superseded by improved conjectures (theorems) and concepts (proof-generated or theoretical concepts) growing out of the method of proofs and refutations. And as theoretical ideas and concepts supersede naive ideas and concepts, theoretical language supersedes naive language. [12, p. 91]

The disagreement of Grünbaum and Shephard with Hilton and Pedersen centers on how to resolve the issues of 'naive' classification in our theoretical language. More precisely, they dispute which theoretical language's vocabulary more appropriately captures 'the' conceptualization of polyhedra lurking behind Euler's original insight. But we need only to acknowledge that no one such language captures all conceptualizations of the object of inquiry to retreat from the dispute and acknowledge that both conceptualizations as reflected in the different choices of words bear mathematical fruit. There is then no need to legislate against alternative conceptualizations by fixing word choices in our theoretical language.

Lakatos' imaginary pupil Omega illustrates the sort of mindset engendering such disputes:

Omega: In the end we shall arrive from naive, accidental, merely nominal classification to the final true, real, classification, to perfect language! [12, p. 92]

The dispute in the American Mathematical Monthly may have been better framed by acknowledging that realizing Omega's dream is both improbable and potentially stifles new research needlessly, and by focusing on the key issue underlying the debate: how change in our choice of words alters our interpretation of results and findings.

As we have seen, refutation by counterexamples depends on the meaning of the terms in question.

If a counterexample is to be an objective criticism, we have to agree on the meaning of our terms.

$[12$, p. 16$]$

As knowledge grows, languages change. [12, p. 93]

Word choices are our looking-glass for mathematical inquiry, but they are not a transparent medium for conceptualizing mathematical subject-matter: our language non-trivially alters our mathematical practices - how we define objects, develop theorems and proofs, and what mathematical facts are noticed (or noticeable). We no longer use words as Euclid or Euler did; it makes little sense to bind our word 'polyhedron' to their historical uses of it, which are in any case quite divergent. Yet tracing the different uses of 'polyhedron' back to Euclid and Euler as we have done here shows how shifting word choices can indicate or inspire shifts in mathematical practices. These shifts in word choice, as we saw in Section 2, can produce new mathematical results about the objects of inquiry. As Lambda says:

Alpha: I propose one single rule instead: Construct rigorous (crystal clear) proofs.

Lambda: ...Cannot 'crystal-clear' thought-experiments lead to paradoxical or even contradictory results?

Alpha: Language is vague, but thought can achieve absolute rigour. [12, p. 52]

We rely on language to show our concepts to others, but language can (and frequently does) imperfectly perform that task for us. We think the argument between Grünbaum and Shephard and Hilton and Pedersen provides an instance of this phenomenon: the word 'polyhedron' historically has given rise to many different conceptualizations of a mathematical subject-matter with a common core of objects, but with divergence in the range of objects falling under that term. Our two pairs of authors have shown that these conceptualizations can be considered as 'rival' ones, even though nothing about them leading to different results makes them 'rivals'. Moreover, these pairs of authors demonstrated that different word choices (embodying different conceptions) lead to different mathematics, showing that mathematicians' word choices in practice demand non-trivial selections.

In short, the encoding of mathematical concepts in language, including our choice of words, is a key component of mathematical practice and meta-mathematical inquiry. It is just as much a part of mathematics as theorem discovery and proof writing. And in the present authors' view, different word choices encoding different mathematics is a boon to mathematical activity - at least so long as our communal understanding of the subject-matter of mathematics remains incomplete. ${ }^{28}$

\footnotetext{
28 We thank our anonymous reviewers for numerous helpful suggestions and criticisms. This work was undertaken with the support of a Luther Rice Fellowship from George Washington University, and we are grateful for that support.
} 


\section{References}

1. Bill Barton, The Language of Mathematics, Mathematics Education Library 46, Springer, 2008.

2. H. S. M. Coxeter, Regular Polytopes, Dover Publications, Inc., 1973.

3. Peter R. Cromwell, Polyhedra, Cambridge University Press, 1997.

4. Euclid, Thomas L. Heath (translator), Euclid's Elements, Green Lion Press, 2010.

5. Leonhard Euler, Christopher Francese and David Richeson (translators), "Proof of Some Notable Properties with Which Solids Encased by Plane Faces are Endowed," Novi Commentarii Academiae Scientiarum Petropolitanae, 4: $72-93,1758$.

6. Christopher Francese and David Richeson, "The Flaw in Euler's Proof of His Polyhedral Formula," American Mathematical Monthly, Vol. 114, No. 4: 286-296, Apr. 2007.

7. Branko Grünbaum, Convex Polytopes, John Wiley \& Sons, Ltd., 1967.

8. Branko Grünbaum, "Are Your Polyhedra the Same as My Polyhedra?" pp. 461-488 in Discrete and Computational Geometry: The Goodman-Pollock Festschrift, B. Aronov, S. Basu, J. Pach, and Sharir, M. (editors), Springer, 2003.

9. Branko Grünbaum, "Graphs of polyhedra; polyhedra as graphs," Discrete Mathematics Volume 307, Issues 3-5: pp. 445-463, 2007.

10. Branko Grünbaum and G. C. Shephard, "A New Look at Euler's Theorem for Polyhedra," American Mathematical Monthly, Vol. 101, Issue 2: 109-128, 1994.

11. Peter Hilton and Jean Pedersen, "Euler's Theorem for Polyhedra: A Topologist and Geometer Respond," American Mathematical Monthly, Vol. 101, Issue 10: 959-962, 1994.

12. Imre Lakatos, John Worrall and Elie Zahar (editors), Proofs and Refutations: The Logic of Mathematical Discovery, Cambridge University Press, 1976.

13. George Lakoff and Rafael Nuñez, Where Mathematics Come From: How The Embodied Mind Brings Mathematics Into Being, Basic Books, 2001.

14. Paolo Mancosu (editor), The Philosophy of Mathematical Practice, Oxford University Press, 2008.

15. David Reed, Figures of Thought, Routledge, 1995.

16. C. Edward Sandifer, How Euler Did It, The Mathematical Association of America, 2007. 